JoÃo Noguelra Neto'

OrLANDO Jorge Martins TORRES ${ }^{2}$

Marilene Oliveira da Rocha Borges

Tarcísio Mota Coelho ${ }^{4}$

ana Gisela Portela de Araújo

CORTES NASCIMENTO

Joel Nicolau Noguera Nunes JúNior ${ }^{6}$

Gabriel LACERDA EsteVES 6

Artigos originais

Palavras-chaves

Sinvastatina/uso terapêutico Endometriose/quimioterapia Ratos Wistar

Keywords

Simvastatin/therapeutic use Endometriosis/drug therapy Rats Wistar

João Nogueira Neto Rua Miragem do Sol, 19, apto. 1.001 - Renascença II CEP 65075-760 - São Lứs/MA Fax: (98) $3231-5010$ E-mili:-inoguiran@ulcombr

Recebido 12/02/2007

Aceito com modificacoōes $03 / 07 / 2007$

\section{Modificações do volume e da histologia de focos de endometriose em ratas tratadas} com sinvastatina

\author{
Changes in the volume and histology of focus of endometriosis \\ in rats treated with sinvastatin
}

Resumo

OBJETIVO: analisar alterações macroscópicas e histológicas que ocorrem com o uso da sinvastatina em endometriose experimental em ratas. MÉTODOS: quarenta ratas da linhagem Wistar foram submetidas à técnica de autotransplante uterino em mesentério. Após três semanas, 24 ratas desenvolveram endometriose experimental grau Ill e foram divididas em dois grupos: Sinvastatina (dado $20 \mathrm{mg} / \mathrm{kg} /$ dia via oral) e Controle (dado cloreto de sódio a 0,9\% na quantidade de $1 \mathrm{~mL} / 100 \mathrm{~g}$ de peso corpóreo via orall, que receberam gavagem durante 14 dias seguido de morte. Os volumes dos implantes foram calculados [ $4 \pi$ (comprimento/2) x (largura/2) x (altura/2)/3] nas intervenções cirúrgicas e após a morte dos animais. Os autotransplantes foram retirados, corados com a hematoxilina-eosina e analisados à microscopia de luz. Foram usados o teste de Mann-Whitney para amostras independentes e o teste de Wilcoxon para amostras relacionadas. Para avaliação histológica, foi usado o teste exato de Fisher, adotando-se nível de significância de 5\%. RESULTADOS: a diferença entre os volumes médios iniciais dos autotransplantes nos dois grupos foi insignificante $(p=1,00)$, e, entre os volumes médios finais, significante $(p=0,04)$. Houve aumento significativo $(p=0,01)$ entre os volumes médios iniciais e finais do Grupo Controle e redução insignificante no Grupo Sinvastatina ( $p=0,95)$. Histologicamente $(p=0,64)$, o Grupo Sinvastatina $(n=9)$ mostrou a parede epitelial moderadamente preservada em sete casos $(77,80 \%)$ e dois casos com camada epitelial bem preservada $(22,2 \%)$, e o Grupo Controle $(n=12)$ com sete casos $(58,30 \%)$ moderadamente preservados e cinco casos $(41,70 \%)$ bem preservados. CONCLUSÕES: a sinvastatina impediu o crescimento dos focos de endometriose experimental. São promissores os estudos com uso da sinvastatina por período mais prolongado.

\section{Abstract}

PURPOSE: to analyze the macroscopic and histological changes that occur with the use of sinvastatin in experimental endometriosis in female rats. METHODS: forty Wistar female rats were submitted to the technique of uterine self-transplant in mesenterium. After three weeks, 24 of them developed experimental endometriosis grade III, and were divided in two groups: one group received sinvastatin orally $(20 \mathrm{mg} / \mathrm{kg} /$ day) and the other (control group) received $0.9 \%$ of sodium chloride orally $(1 \mathrm{~mL} / 100 \mathrm{~g}$ of body weight/day). Both groups received gavage for 14 days, followed by death. The implant volume was calculated [ $4 \pi($ lenght/2) $\times($ width/2) $\times($ height/2)/3] at the surgical intervention and after the animal's death. The self-transplants were removed, dyed with hematoxylin-eosin and analyzed by light microscopy. The Mann-Whitney's test was used in the independent samples and the Wilcoxon's test for the related samples. The Fisher's exact test was used for the histological evaluation, with a significance level of 5\%. RESULTS: the difference between groups of the initial average volumes of the self-transplants was not significant $(p=1.00)$, but became significant for the final average volumes $(p=0.04)$. There was a significant increase $(p=0.01)$ between the initial and final average volumes in the control group, and a no significant decrease in the sinvastatin group ( $p=0.95)$. Histologically, the sinvastatin group $(n=9)$ presented seven cases $(77.8 \%)$ of moderately preserved and two cases $(22.2 \%)$ of well preserved epithelial wall, while the control group $(n=12)$ presented seven cases $(58.3 \%)$ of moderately preserved and five cases $(41.7 \%)$ of well preserved epithelial wall. CONCLUSIONS: sinvastatin prevented the growth of experimental endometriosis. Studies with sinvastatin for longer periods are promising

Centro de Ciências da Saúde e Laboratório de Cirurgia Experimental da Universidade Federal do Maranhão - UFMA - São Luís (MA), Brasil.

'Pós-Graduando do Programa de Mestrado em Ciências da Saúde da Universidade Federal do Maranhão - UFMA - São Luís (MA), Brasil.

Livre-Docente, Professor do Departamento de Medicina II da Universidade Federal do Maranhão - UFMA - São Luís (MA), Brasil. 3 Professora Adjunta do Departamento de Ciências Fisiológicas da Universidade Federal do Maranhão - UFMA - São Luís (MA), Brasil. ${ }_{4}$ Professor Adjunto do Departamento de Medicina III da Universidade Federal do Maranhão - UFMA - São Luís (MA), Brasil.

5 Patologisła do Hospital Universitário Presidente Dutra da Universidade Federal do Maranhão - UFMA - São Luís (MA), Brasil.

${ }^{\circ}$ Acadêmico do Curso de Medicina da Universidade Federal do Maranhão - UFMA - São Luís (MA), Brasil. 


\section{Introdução}

A endometriose é uma doença caracterizada pela presença de tecido estrutural e funcionalmente semelhante ao tecido endometrial fora da cavidade uterina. O tratamento pode ser clínico e/ou cirúrgico, sendo que as terapêuticas medicamentosas são preferencialmente aquelas que interferem no ciclo menstrual, induzindo pseudogravidez, pseudomenopausa ou anovulação crônica, proporcionando melhora clínica, porém seus efeitos não são curativos, o que justifica a busca de novos tratamentos ${ }^{1}$.

As pesquisas experimentais sobre endometriose ganharam grande impulso após estudos experimentais que descreveram a técnica cirúrgica do autotransplante de tecido uterino em ratas para desenvolver endometriose experimental peritoneal ${ }^{2}$. Desde então, várias drogas objetivando o tratamento da endometriose foram testadas naquele modelo ${ }^{3,4}$.

Em estudo sobre o efeito individual e combinado da triptorelina e gestrinona em endometriose experimental em ratas, os autores apresentaram classificação macroscópica dos autotransplantes, baseada fundamentalmente na formação cística do autotransplante. Essa classificação macroscópica tornou-se parâmetro para avaliar o sucesso ou não de tratamentos com medicamentos experimentais ${ }^{5}$.

A classificação histológica da endometriose experimental mais usada, apesar de subjetiva, visa avaliar de forma semiquantitativa a persistência de células epiteliais dos autotransplantes pós-tratamento experimental, em que as camadas podem estar ausentes, pobres, moderadas ou bem preservadas ${ }^{6}$.

As estatinas representam classe de drogas que efetivamente podem baixar os níveis séricos de colesterol, sendo largamente usadas no tratamento de hipercolesterolemia, por meio da inibição competitiva da 3-hidroxi-3-metilglutaril coenzima A redutase, enzima da via de síntese do colesterol no fígado e outros tecidos ${ }^{7}$. Estudos experimentais mostram que essas drogas diminuem efetivamente processos inflamató$\operatorname{rios}^{8,9}$, têm efeito inibitório na multiplicação celular ${ }^{10}$ e modulam negativamente a proliferação endometrial e a angiogênese ${ }^{11}$. A presença de fatores de angiogênese é observada em maior quantidade na pelve de mulheres com endometriose ${ }^{12}$.

O estresse oxidativo contribui na etiopatogenia da endometriose $^{13}$, uma vez que as mulheres com endometriose apresentam níveis elevados de autoanticorpos marcadores do estresse oxidativo ${ }^{14}$. As estatinas possuem atividade antioxidante intrínseca, sendo a sinvastatina citada como a mais efetiva ${ }^{15}$.
Diante desses aspectos, objetivou-se analisar, sob aspectos macroscópicos e histológicos, a repercussão do emprego da sinvastatina via oral sobre endometriose experimental induzida por meio de autotransplante em ratas.

\section{Métodos}

Este estudo foi experimental, realizado durante o período compreendido entre junho e setembro de 2006, com amostra de 40 ratas da linhagem Wistar (Rattus norvegicus albinus), adultas, fêmeas, virgens, pesando entre 180 e 250 g, com 60 dias de vida, cedidas pela central do Biotério da Universidade Federal do Maranhão (UFMA).

A pesquisa foi desenvolvida no Laboratório de Cirurgia Experimental do Hospital Universitário da UFMA. Foram respeitadas a legislação brasileira para o uso de animais de experimentação (Lei Federal n 6.638/1979) e as normas do Colégio Brasileiro de Experimentação Animal (COBEA), instituição filiada ao International Council for Laboratory Animal Science. O estudo foi aprovado pelo Comitê de Ética e Experimentação Animal (CEEA-UEMA), protocolo 05/2006.

Os animais foram agrupados em número de cinco por gaiola de polipropileno com tampa de grade metálica inoxidável, medindo $46 \mathrm{~cm} \mathrm{x} 31 \mathrm{~cm} \mathrm{x} 16 \mathrm{~cm}$, com fundo forrado com papel, que era trocado a cada 48 horas. Os animais foram divididos em oito grupos mantidos em condições ambientais constantes, recebendo ração para ratos (Purina ${ }^{\circledR}$, São Paulo, Brasil) e água ad libitum durante sete dias de adaptação, com controle de ruídos, temperatura de $22 \pm 2^{\circ} \mathrm{C}$, umidade relativa 40 a $60 \%$ e ciclos claro/escuro de 12/12 horas. Paralelamente, foram realizadas coleta e análise de esfregaço vaginal diariamente. Somente aquelas ratas que apresentaram quatro ou mais ciclos estrais a cada cinco dias foram incluídas no estudo ${ }^{16}$.

Após jejum de 12 horas, os animais foram pesados com balança eletrônica (Marte ${ }^{\circledR}$, modelo A500, 110-200, indústria brasileira) e anestesiados com mistura de xilazina $2 \%$ e ketamina $5 \%$ na proporção de $1: 1$, na dose de 0,2 $\mathrm{mL}$ para cada $100 \mathrm{~g}$ de peso, via intramuscular, com agulha hipodérmica de $13 \mathrm{~mm}$ x 4,5 mm (Becton Dickinson, Paraná, Brasil), na borda posterior da coxa esquerda ${ }^{17}$. Os animais foram considerados anestesiados na ausência de reflexos corneanos e interdigitais. Foi realizada a epilação manual da região abdominal caudal do animal com área de $5 \mathrm{~cm} \mathrm{x} 4 \mathrm{~cm}$, anti-sepsia com polivinil-pirrolidona-iodo (Povidine Tópico ${ }^{\circledR}$, São Paulo, SP) e colocação de campo fenestrado. Nesta fase, não foi avaliado o ciclo estral. 
Uma incisão mediana de $3 \mathrm{~cm}$ foi realizada com bisturi de lâmina n. ${ }^{\circ} 15$, acometendo pele, plano músculo-aponeurótico e peritôneo, seguido de identificação dos órgãos intracavitários e exposição do útero, anexos e mesentério. O procedimento cirúrgico seguiu com identificação, apreensão, ligadura e secção do terço médio do corno uterino esquerdo usando fio de náilon 6-0 (Mononylon ${ }^{\circledR}$, Ethicon, São Paulo, Brasil) com três pontos simples e hemostasia. Concomitantemente, foi realizado fatiamento da peça extraída do útero em cortes longitudinais, seguido de cortes transversais, formando retalhos com medidas de $4 \mathrm{~mm}$ x $4 \mathrm{~mm}$, em meio líquido com Ringer lactato ${ }^{\circledR}$, sendo transpassado ponto



Figura 1 - Fotomicrografia mostrando retalho com medidas de $4 \mathrm{~mm} \times$ $4 \mathrm{~mm}$ do corno uterino esquerdo com fio de náilon 6-0 na superfície serosa após autotransplante no mesentério de rata Wistar, deixando a superfície mucosa do retalho voltada para a luz da cavidade peritoneal.



Figura 2 - Fotomicrografia mostrando autotransplante com diâmetro maior que 4,5 mm e classificado como grau III de Quereda et $\mathrm{al}^{5}$. com fio de náilon 6-0 na região central da superfície serosa do retalho e mantido reparado.

O retalho foi autotransplantado para o mesentério, próximo ao vaso que irriga o cecum, mantendo-se a superfície serosa em contato com o mesentério e a superfície endometrial voltada para a luz da cavidade peritoneal. A fixação foi confeccionada com um ponto simples do retalho reparado acima. A cavidade peritoneal ficou coberta com gaze embebida com cloreto de sódio a $9 \%$ durante todo o procedimento (Figura 1 ).

A síntese da parede abdominal foi realizada em dois planos de sutura contínua acometendo o plano músculo-aponeurótico e a pele com fio de náilon 5-0. Os animais permaneceram no laboratório por um período de 21 dias. Após esse período, as ratas foram reoperadas; realizou-se o inventário da cavidade peritoneal com identificação e medida do foco de autotransplante com paquímetro digital, seguido do cálculo do volume $[4 \pi$ (comprimento/2) x (largura/2) x (altura/2)/3 $]^{18}$. Foi usado o Sistema de Classificação dos Implantes em Grau de Crescimento de acordo com Quereda et al. ${ }^{5}$, e somente aquelas que desenvolveram grau de crescimento III, ou seja, com diâmetro $\geq 4,5 \mathrm{~mm}$, seguiram no estudo (Figura 2).

Após o procedimento cirúrgico, as ratas classificadas em grau III foram identificadas e divididas em dois grupos de 12 ratas, distribuídas aleatoriamente. O Grupo Controle recebeu gavagem com solução de cloreto de sódio a 0,9\% na quantidade de $1 \mathrm{~mL} / 100 \mathrm{~g}$ de peso corpóreo do animal, e o Grupo Sinvastatina recebeu gavagem com $20 \mathrm{mg} / \mathrm{kg}$ de peso corpóreo de sinvastatina (Galena, São Paulo, Brasil). Os dois grupos receberam gavagem uma vez ao dia em torno das 19 horas, com pesagem diária das ratas para correção da dose, por um período de 14 dias.

Os animais de cada grupo (Grupos Controle e Sinvastatina) foram mortos no $14^{\circ}$ dia após o início das gavagens. Os animais foram pesados e colocados em vaporizador de éter até a morte, caracterizada por parada respiratória e ausência completa de reflexos ${ }^{19}$. A seguir, toda a área ventral abdominal foi epilada, seguido da realização de duas incisões transversais: acima, margeando o rebordo costal, e abaixo, margeando os ossos pélvicos, seguindo com uma terceira incisão longitudinal lateral esquerda, unindo as incisões transversais, expondo completamente a cavidade peritoneal.

Realizou-se o inventário da cavidade peritoneal e anotaram-se as medidas do volume do autotransplante, seguido de sua excisão. A peça retirada foi limpa com cloreto de sódio a $0,9 \%$ e acondicionada em recipiente contendo formol tamponado a $10 \%$, 
recebendo identificação. A avaliação histológica foi realizada no Serviço de Anatomia Patológica do Hospital Universitário da UFMA. As lâminas foram coradas pela hematoxilina-eosina. A análise histológica foi realizada por uma única patologista, que levou em consideração a persistência de células epiteliais dos autotransplantes semiquantitativamente e classificou em grau III a parede bem-preservada; grau II, a parede epitelial moderadamente preservada, com infiltração de leucócitos; grau I, a parede epitelial pobremente preservada, com uma célula epitelial ocasional e grau zero, sem camada epitelial ${ }^{6}$.

A análise estatística utilizou o programa Biostat 3.0 em Windows XP, em que o nível de significância $(\alpha)$ utilizado para se rejeitar a hipótese de nulidade foi de $5 \%(\mathrm{p}<0,05)$. Aplicado o teste de normalidade de Shapiro-Wilk, os dados não apresentaram distribuição normal; sendo assim, foi usado o teste de Mann-Whitney para amostras independentes e o teste de Wilcoxon para amostras relacionadas. Para comparação dos achados histológicos, foi usado o teste exato de Fisher.

\section{Resultados}

Houve três óbitos de ratas no Grupo Sinvastatina por bronco-aspiração, restando uma amostra experimental de nove ratas. As médias dos volumes iniciais em $\mathrm{mm}^{3}$ do Grupo Controle e do Grupo Sinvastatina foram, respectivamente, $38,1 \mathrm{~mm}^{3}$ e $48,9 \mathrm{~mm}^{3}(\mathrm{p}=1,0)$. Após o período de duas semanas, com administração da sinvastatina e cloreto de sódio, as médias volumétricas finais foram, respectivamente, $75,7 \mathrm{~mm}^{3}$ e $45,3 \mathrm{~mm}^{3}$, com diferença significante $(\mathrm{p}=0,04)$ (Tabela 1$)$.

A comparação das médias volumétricas em $\mathrm{mm}^{3}$ do Grupo Controle inicial e após duas semanas de gavagem com solução fisiológica mostrou $38,1 \mathrm{~mm}^{3}$ e $75,7 \mathrm{~mm}^{3}$, respectivamente $(p=0,01)$. No Grupo Sinvastatina, a média volumétrica inicial foi de $48,9 \mathrm{~mm}^{3}$ e final de $45,3 \mathrm{~mm}^{3}$, mostrando média volumétrica semelhante $(\mathrm{p}=0,95)$; portanto, no grupo tratado, o volume dos implantes ficou estável, enquanto no Grupo Controle houve aumento significante (Tabela 1).

$\mathrm{Na}$ avaliação histológica, quanto à preservação da camada epitelial $(\mathrm{p}=0,64)$, em todas as amostras houve preservação da camada epitelial; no total de nove ratas do Grupo Sinvastatina $(n=9)$ houve sete casos $(77,80 \%)$ com a camada moderadamente preservada e dois casos $(22,20 \%)$ com a camada epitelial bem preservada. O Grupo Controle $(\mathrm{n}=12)$ apresentou sete casos $(58,30 \%)$ com a camada moderadamente preservada e cinco casos $(41,70 \%)$ com a camada epitelial bem preservada. As camadas bem preservadas (Figura 3) apresentaram a célula epitelial com citoplasma bem desenvolvido e as camadas moderadamente preservadas mostraram uma célula com citoplasma bastante reduzido (Figura 4).

\section{Discussão}

A endometriose é uma doença cuja terapia ideal ainda está por vir. As drogas mais usadas atualmente, que são os progestagênios, danazol, anticoncepcionais orais e análogos de GnRH, têm resultados benéficos nos episódios de dores, mas interferem na fertilidade e a interrupção do uso é sucedida de recorrência da endometriose ${ }^{20}$.

Várias drogas vêm sendo pesquisadas em estudos experimentais como opção terapêutica da endometriose. As alternativas para supressão estrogênica são os inibidores de aromatase e os moduladores seletivos de receptores estrogênicos. Outros são os imunomoduladores, agentes antiinflamatórios ${ }^{21}$ e a vacina de $\mathrm{BCG}^{22}$, porém, o uso de estatinas é pouco citado na literatura ${ }^{11,24-26}$. Paralelamente, a procura por drogas que possuem efeitos contrários ao desenvolvimento da endometriose nos permitiu chegar até as estatinas, por possuírem efeitos antiinflamatórios, principalmente pela interferência na produção de citoquinas pró-inflamatórias ${ }^{27}$, efeitos inibitórios na multiplicação celular ${ }^{10}$ e modulação negativa na proliferação celular e na angiogênese ${ }^{11}$. Estudo constatou que ativadores de angiogênese são mais comuns em fluido peritoneal de mulheres com endometriose que em Grupo Controle ${ }^{12}$ e, por fim, que o efeito antioxidante das estatinas, dando destaque à sinvastatina, deu suporte para se acreditar que poderiam ser pesquisadas como possíveis drogas úteis no combate à endometriose ${ }^{13}$.

$O$ autotransplante da endometriose experimental permitiu o uso experimental de várias drogas no combate à endometriose ${ }^{2}$. Exemplo marcante é o estudo ${ }^{18}$ que analisou o efeito do inibidor de aromatase YM511 em endometriose experimental de ratas e observou,

Tabela 1 - Característica e resultado de grupos de ratas tratadas com sinvastatina ( $20 \mathrm{mg} / \mathrm{kg} /$ dia) e cloreto de sódio (1 mL/100 g de peso corpóreo) via oral durante 14 dias, mostrando que no grupo tratado o volume dos implantes ficou estável, enquanto no Grupo Controle houve aumento significante.

\begin{tabular}{lccc}
\hline Grupo & $\begin{array}{c}\text { Média do volume } \\
\text { inicial }\end{array}$ & $\begin{array}{c}\text { Média do volume } \\
\text { final }\end{array}$ & $\mathbf{p}^{*}$ \\
\hline Sinvastatina & $48,98 \mathrm{~mm}^{3}$ & $45,36 \mathrm{~mm}^{3}$ & 0,95 \\
Controle $p^{* *}$ & $38,10 \mathrm{~mm}^{3}$ & $75,70 \mathrm{~mm}^{3}$ & 0,01 \\
& 1,00 & 0,40 & \\
\hline
\end{tabular}

*Teste de Wilcoxon para amostras relacionadas, com $\mathrm{p}<0,05$ para rejeitar a hipótese de nulidade na comparação das médias dos volumes iniciais e finais dentro dos Grupos Sinvastatina e Controle.

**Teste de Mann-Whitney para amostras indepedentes, com $\mathrm{p}<0,05$ para rejeitar a hipótese de nulidade na comparação dos volumes médios iniciais entre os Grupos Sinvastatina e Controle, e volumes médios finais entre esses grupos. 


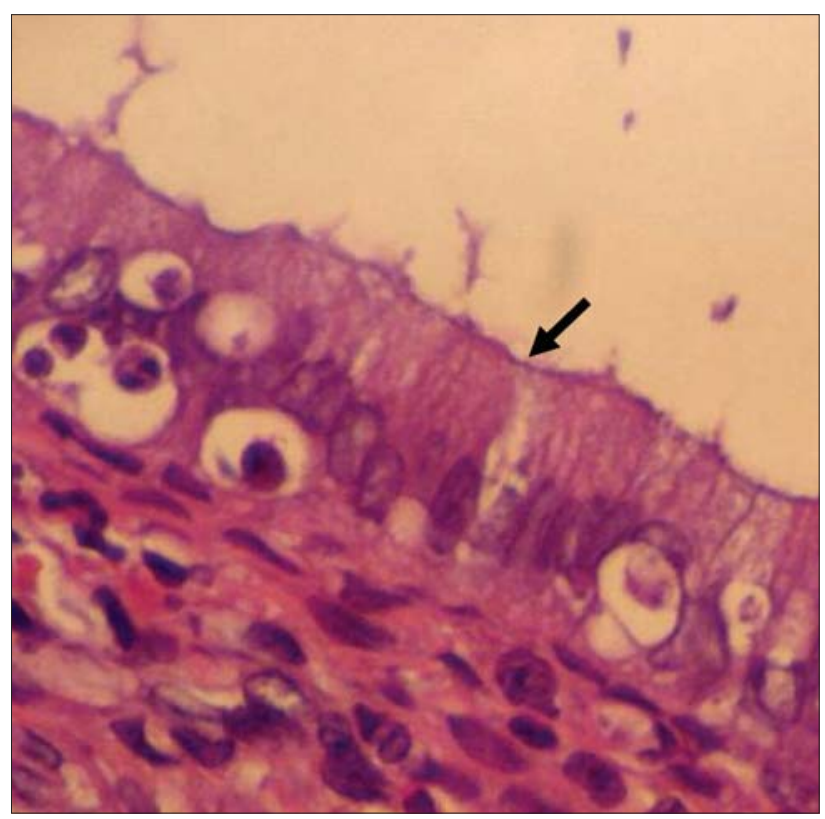

Figura 3 - Fotomicrografia de célula epitelial bem-preservada, com citoplasma (seta) bem desenvolvido (40X) do grupo de ratas tratadas com cloreto de sódio (1 mL/100 g de peso corpóreo) via oral durante 14 dias.

no Grupo Controle, um aumento do volume médio de $33,0 \mathrm{~mm}^{3}$ para $41,0 \mathrm{~mm}^{3}$. Comparando com nosso estudo, observamos aumento do volume médio do Grupo Controle inicial de $38,1 \mathrm{~mm}^{3}$ para $75,7 \mathrm{~mm}^{3}$ no final, mostrando um maior crescimento em nossa amostra, além do menor tempo de observação: 14 dias, contra 21 daquele estudo. Os grupos experimentais desse autor usaram YM511 nas doses de 0,04, 0,2 e $1 \mathrm{mg} / \mathrm{kg}$ e apresentaram redução volumétrica dos autotransplantes significante quando comparados com o Grupo Controle e ao grupo que foi submetido a ooforectomia bilateralmente do mesmo estudo. No nosso estudo, não tivemos o grupo de ooforectomia, nem várias doses, apenas uma dose de $20 \mathrm{mg} / \mathrm{kg} / \mathrm{dia}$ de sinvastatina no Grupo Experimental, o que, na comparação dos volumes finais entre os Grupos Controle e Experimental, mostrou redução volumétrica significante, e, na comparação dos volumes inicial e final do Grupo Experimental, não houve evolução da endometriose experimental.

A dose de $20 \mathrm{mg} / \mathrm{kg} /$ dia utilizada em nossa pesquisa foi baseada em estudo que constatou atenuação de lesões isquêmicas cerebrais por hipóxia em ratos recém-nascidos, por meio das reduções de resposta inflamatória, ativação de caspase-3 e da morte celular por apoptose ${ }^{9}$. Esses autores fizeram uso da mesma posologia de sinvastatina a $20 \mathrm{mg} / \mathrm{kg}$ por via subcutânea por período de duas semanas. A escolha da via oral por gavagem em nossa pesquisa foi pelo fato de

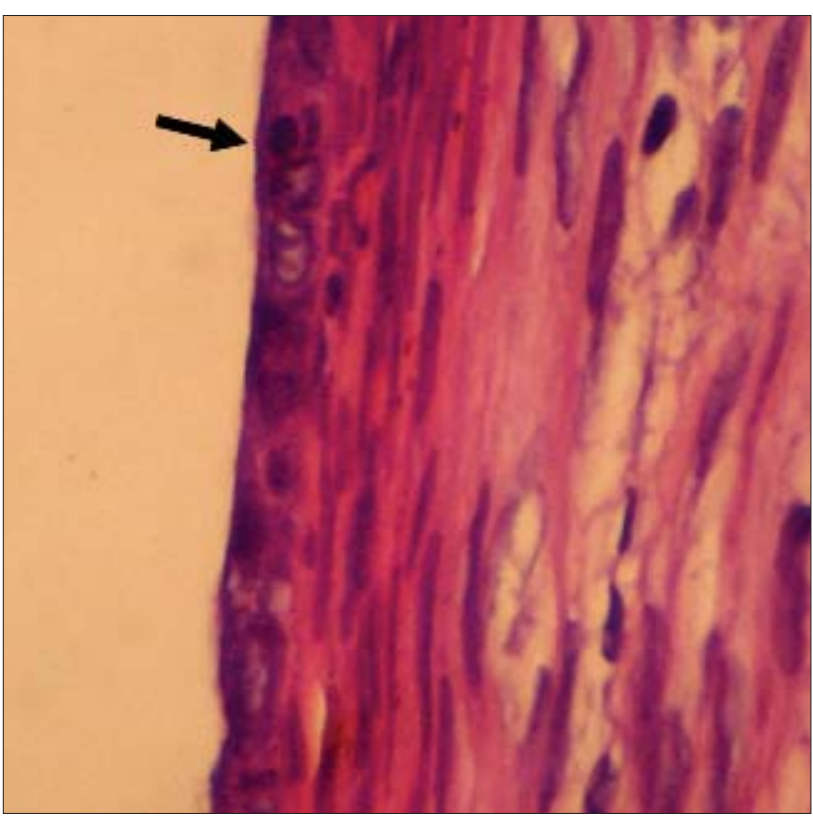

Figura 4 - Fotomicrografia de célula epitelial moderadamente preservada com citoplasma (seta) pouco desenvolvido (40X) do grupo de ratas tratadas com sinvastatina $(20 \mathrm{mg} / \mathrm{kg} / \mathrm{dia}$ ) via oral durante 14 dias.

ser essa via mais comumente utilizada para o uso das estatinas na prática diária.

O uso de estatina como potencial agente terapêutico no tratamento da endometriose é sugerido em estudo que avaliou o efeito dessa droga na interleucina- $8 \mathrm{em}$ células e estroma endometrial de mulheres com patologia benigna in vitro, em que foram utilizadas mevastatina e sinvastatina nas doses de 3,10 e $30 \mu \mathrm{M}$, após exposição de 24 horas. Ambas as drogas apresentaram redução significante da interleucina-8 na dosagem de $30 \mu \mathrm{M}^{24}$. O efeito da inibição do crescimento das células e estroma endometrial exposto à mevastatina e sinvastatina seria devido à inibição da síntese de $\mathrm{DNA}^{24}$.

$\mathrm{O}$ uso de atorvastatina em modelo experimental de endometriose foi avaliado em quatro grupos: Grupo I (baixa dose de atorvastatina, oito ratas) recebeu $0,5 \mathrm{mg} / \mathrm{kg} / \mathrm{dia}$ via oral; Grupo II (alta dose de atorvastatina, dez ratas) recebeu $2,5 \mathrm{mg} / \mathrm{kg} / \mathrm{dia}$ via oral; Grupo III (acetato de leuprolide, nove ratas), recebeu $1 \mathrm{mg} / \mathrm{Kg} /$ subcutânea e Grupo IV (Grupo Controle sem medicação), sendo avaliados o nível peritoneal do fator de crescimento endotelial vascular, a macroscopia e a histologia após o período de 21 dias. A regressão do nível do fator de crescimento endotelial vascular e dos implantes foi significante nos Grupos II e III ${ }^{26}$. Em nosso estudo, usamos apenas uma dose (sinvastatina, $20 \mathrm{mg} / \mathrm{kg} / \mathrm{dia}$, via oral) pelo período de 14 dias, com redução de $48,98 \mathrm{~mm}^{3}$ para $45,98 \mathrm{~mm}^{3}$, contra $41,2 \mathrm{~mm}^{3}$ para $13,1 \mathrm{~mm}^{3}$ do Grupo II e de $43,0 \mathrm{~mm}^{3}$ 
para 50,5 $\mathrm{mm}^{3}$ no Grupo I, e no Grupo Controle, não significante, contrastando com nosso estudo, que foi significante. Apesar da forma de medição do volume diferente, essa comparação sugere a necessidade de um período maior de exposição, outras dosagens e controle sem medicação.

Histologicamente, a atrofia da camada epitelial poderia acontecer devido à maior pressão intravesical, porém, a menor média volumétrica do Grupo Experimental e a menor quantidade proporcional de epitélio bem preservado afastaram essa hipótese. $\mathrm{O}$ maior comprometimento epitelial, apesar de insignificante, e a maior redução volumétrica média do Grupo Experimental abrem caminho para a suposição de que a sinvastatina deva ser mais investigada como possível opção no tratamento da endometriose.

O uso dos imuno-moduladores loxoribina e levamisole foi analisado em endometriose experimental de ratas, devido a evidências que comprovam o envolvimento de fatores imunológicos na fisiopatologia e etiologia da endometriose ${ }^{11}$. Esse estudo mostrou, na avaliação histológica quanto à preservação da camada epitelial, diferença significante entre a loxoribina e

\section{Referências}

1. Oliver DL, Pritts EA. Treatment of endometriosis. N Engl J Med. 2001;345(4):266-75.

2. Vernon MW, Wilson EA. Studies on the surgical induction of endometriosis in the rat. Fertil Steril. 1985;44(5):684-94.

3. Fortin $M$, Lépine $M$, Pagé $M$, Osteen $K$, Massie $B$, Hugo PE, et al. An improved mouse model for endometriosis allows noninvasive assessment of lesion implantation and development. Fertil Steril. 2003;80 Suppl 2:832-8.

4. Beliard A, Noel A, Goffin F, Frankenne FE, Foidart JM. Role of endocrine status and cell type in adhesion of human endometrial cells to the peritoneum in nude mice. Fertil Steril. 2002;78(5):973-8.

5. Quereda F, Barroso J, Acien P. Individual and combined effects of triptoreline and gestrinone on experimental endometriosis in rat. Eur J Obstet Gynecol Reprod Biol. 1996;67(1):35-40.

6. Keenan JA, Williams-Boyce PK, Massey PJ, Chen TT, Caudle MR, Bukovsky A. Regression of endometrial explants in a rat model of endometriosis treated with the immune modulators loxoribine and levamisole. Fertil Steril. 1999;72(1):135-41.

7. Weitz-Schmidt G. Statins as anti-inflammatory agents. Trends Pharmacol Sci. 2002;23(10):482-6.

8. Barsante MM, Roffe E, Yokoro CM, Tafuri WL, Souza DG, Pinho $V$, et al. Anti-inflammatory and analgesic effects of atorvastatin in a rat model of adjuvant-induced arthritis. Eur J Pharmacol. 2005; $516(3): 282-9$.

9. Carloni S, Mazzoni E, Cimino M, De Simoni MG, Perego C, Scopa C, et al. Simvastatin reduces caspase-3 activation and inflammatory markers induced by hypoxia-ischemia in the newborn rat. Neurobiol Dis. 2006;21(1):119-26. o Grupo Controle; também houve diferença significante entre loxoribina e levamisol e não significante entre o Grupo Controle e Levamisol ${ }^{6}$. $\mathrm{Na}$ avaliação histológica, após a exposição com atorvastatina, houve maior comprometimento da camada epitelial nos grupos com alta dose de atorvastatina $(2,5 \mathrm{mg} / \mathrm{kg} / \mathrm{dia})$ e acetato de leuprolide ${ }^{26}$. Nosso estudo mostrou, na sua análise histológica, maior comprometimento da camada epitelial insignificante no Grupo Sinvastatina, sugerindo a necessidade de um período de maior exposição à sinvastatina.

Como conclusão, os dados mostram uma manutenção dos volumes médios iniciais e finais dos autotransplantes do Grupo Sinvastatina. O Grupo Controle apresentou aumento volumétrico de forma significante. A comparação dos volumes médios finais dos dois grupos mostra uma diferença significante, apontando que a sinvastatina interferiu no desenvolvimento da endometriose experimental e traz a necessidade de exposição dos autotransplantes à sinvastatina por período mais prolongado e análise mais profunda para identificar o mecanismo de ação dessa droga na fisiopatologia da endometriose experimental.
10. Corsini A, Raiteri M, Soma MR, Bernini F, Fumagalli R, Paoletti $R$. Pathogenesis of atherosclerosis and the role of 3-hydroxy-3methylglutaryl coenzime A reductase inhibitors. Am J Cardiol. 1995;76(2):21A-28A.

11. Esfandiari N, Ai J, Khazaei M, Javed M, Gotlieb L, Casper RF. Effect of a statin on an in vitro model of endometriosis. Fertil Steril. 2005;84 Suppl 1:S123.

12. Taylor RN, Lebovic DI, Mueller MD. Angiogenic factors in endometriosis. Ann N Y Acad Sci. 2002;955:89-100.

13. Van Langendonckt A, Casanas-Roux F, Donnez J. Oxidative stress and peritoneal endometriosis. Fertil Steril. 2002;77(5):861-70.

14. Shanti A, Santanam N, Morales AJ, Parthasarathy S, Murphy AA. Autoantibodies to markers of oxidative stress are elevated in women with endometriosis. Fertil Steril. 1999;71(6):1 11 15-8.

15. Franzoni F, Quinones-Galvan A, Regoli F, Ferrannini E, Galetta F. A comparative study of the in vitro antioxidant activity of statins. Int J Cardiol. 2003;90(2-3):317-21.

16. Marcondes FK, Bianchi FJ, Tanno AP. Determination of the estrous cycle phases of rats: some helpful considerations. Braz J Biol. 2002;62(4A):609-14.

17. Braz F, Rahal SC, Rocha NS, Taga E, Biasi F. Emprego de matriz óssea orgânica bovina e hidroxiapatita no reparo de defeito induzido em crânio de ratos. Acta Cir Bras. 2003;18(1):19-24.

18. Kudoh $M$, Susaki $Y$, Ideyama $Y$, Nanya T, Mori $M$, Shikama $H$. Inhibitory effects of a novel aromatase inhibitor, YM511, on growth of endometrial explants and insulin-like growth factor-l gene expression in rats with experimental endometriosis. J Steroid Biochem Mol Biol. 1997;63(1-3):75-80. 
19. Brito MVH, Brito NMB, Almeida AJB, Santos MRLC. Vaporizador artesanal de éter para cirurgia experimental em ratos. Acta $\mathrm{Cir}$ Bras. 1998; 13(1):3-5.

20. Dogan E, Saygili U, Posaci C, Tuna B, Caliskan S, Altunyurt S, et al. Regression of endometrial explants in rats treated with the cyclooxygenase-2 inhibitor rofecoxib. Fertil Steril. 2004;82 Suppl 3:1115-20.

21. Vignali M, Infantino M, Matrone R, Chiodo I, Somigliana E, Busacca $M$, et al. Endometriosis: novel etiopathogenetic concepts and clinical perpectives. Fertil Esteril. 2002;78(4):665-78.

22. Gul A, Yasar T, Ugras S. BCG vaccination to prevent implantation of endometriosis: an experimental study in rats. Eur J Obstet Gynecol Reprod Biol. 2001;98(2):209-12.
23. Piotrowski PC, Kwintkiewicz J, Rzepczynska IJ, Seval Y, Cakmak $H$, Arici $A$, et al. Statins inhibit growth of human endometrial stromal cell indepedendently of cholesterol availability. Biol Reprod. 2006;75(1):107-11.

24. Seval Y, Luk J, Kwintkiewicz J, Duleba AJ, Arici A. Statins downregulate interleukin-8 expression in endometrial stromal cells in vitro. Fertil Steril. 2004;82 Suppl 2:S167-S168.

25. Oktem M, Esinler I, Eroglu D, Haberal N, Bayraktar N, Zeyneloglu $\mathrm{HB}$. High-dose atorvastatin causes regression of endometriotic implants: a rat model. Hum Reprod. 2007;22(5):1474-80.

26. Kwak BR, Mulhaupt F, Mach F. Atherosclerosis: anti-inflammatory and immunomodulatory activities of statins. Autoimmunity Rev. $2003 ; 2(6): 332-8$. 